Open Access

\title{
Outcomes of a rapid refeeding protocol in Adolescent Anorexia Nervosa
}

Sloane Madden ${ }^{1,2,3^{*}}$, Jane Miskovic-Wheatley ${ }^{1,3}$, Simon Clarke ${ }^{4,5}$, Stephen Touyz ${ }^{6}$, Phillipa Hay ${ }^{7}$ and Michael R Kohn ${ }^{1,3,4,5}$

\begin{abstract}
Background: The impact of severe malnutrition and medical instability in adolescent Anorexia Nervosa (AN) on immediate health and long-term development underscores the need for safe and efficient methods of refeeding. Current refeeding guidelines in AN advocate low initial caloric intake with slow increases in energy intake to avoid refeeding syndrome. This study demonstrates the potential for more rapid refeeding to promote initial weight recovery and correct medical instability in adolescent AN.
\end{abstract}

Methods: Seventy-eight adolescents with AN (12-18 years), hospitalised in two specialist paediatric eating disorder units, for medical instability (bradycardia, hypotension, hypothermia, orthostatic instability and/or cardiac arrhythmia) were followed during a 2.5 week admission. Patients were refed using a standardised protocol commencing with 24-72 hours of continuous nasogastric feeds (ceased with daytime medical stability) and routine oral phosphate supplementation, followed by nocturnal feeds and a meal plan of 1200-2400 kcal/day aiming for a total caloric intake of $2400-3000 \mathrm{kcal} /$ day. Along with indicators of medical stability, weight, phosphate and glucose levels were recorded.

Results: All patients gained weight in week one $(M=2.79 \mathrm{~kg}, S D=1.27 \mathrm{~kg})$ and at subsequent measurement points with an average gain of $5.12 \mathrm{~kg}(S D=2.96)$ at 2.5 weeks. No patient developed hypophosphatemia, hypoglycaemia, or stigmata of the refeeding syndrome.

Conclusions: The refeeding protocol resulted in immediate weight gain and was well tolerated with no indicators of refeeding syndrome. There were no significant differences in outcomes between the treatment sites, suggesting the protocol is replicable.

Trial registration: Australian Clinical Trials Register number: ACTRN012607000009415

Keywords: Anorexia Nervosa, Eating disorder, Inpatient treatment, Refeeding syndrome, Adolescents, Nasogastric refeeding, Rapid refeeding

\section{Background}

The impact of severe malnutrition in adolescent Anorexia Nervosa (AN) on short-term health and longterm growth demands safe and efficient means for nutritional and weight recovery [1]. This is particularly so in adolescents with medical instability (bradycardia, hypotension, hypothermia, orthostatic instability and cardiac arrhythmia) [2,3]. Increasingly, such patients are admitted to paediatric wards for medical stabilisation,

\footnotetext{
* Correspondence: sloane.madden@health.nsw.gov.au

'Eating Disorder Service, The Sydney Children's Hospitals Network,

Westmead Campus, Locked Bag 4001, Westmead 2145, NSW, Australia

${ }^{2}$ Discipline of Psychiatry, Faculty of Medicine, The University of Sydney,

Sydney, Australia

Full list of author information is available at the end of the article
}

initiation of refeeding and eating disorder treatment [4]. Current refeeding guidelines are inconsistent, lacking in empirical evidence, and do not adequately address physiological requirements for children and adolescents with AN [5].

Over the last two decades, guidelines for the nutritional management of AN have been published by a range of professional organisations including the Society of Adolescent Health and Medicine [3]; the National Institute for Clinical Excellence [6] and the American Psychiatric Association [1]. These current guidelines recommend an initial refeeding rate of between 10 and $40 \mathrm{kcal} / \mathrm{kg}$ per day or between $20-80 \%$ of total daily requirements, with slow caloric increases. Debate is ongoing as to the 
recommended rate of weight gain in inpatient settings with targets ranging from 500 to $1,400 \mathrm{~g}$ per week $[1,7]$. In all cases, the initial caloric prescription is below daily resting energy requirements with only slow increases recommended.

Current refeeding guidelines aim to avoid the refeeding syndrome principally by restricting the intake of calories. The refeeding syndrome is the occurrence of hypophosphatemia, cardiac events, neurological concerns and/or sudden death of patients within two weeks of commencing refeeding [8-15]. Despite the intent, current guidelines have not eliminated the occurrence of refeeding syndrome. A review of the literature by O'Connor and Goldin [14] identified 23 patients who developed refeeding syndrome despite slow rates of refeeding. The average rate of refeeding in these patients was $27 \mathrm{kcal} / \mathrm{kg} /$ day, well below total daily energy requirements. Of note, all patients were less than $70 \%$ of expected body weight for age, height and gender and all developed hypophosphatemia. They concluded individuals treated according to current guidelines might still develop the refeeding syndrome, with the risk not linked to caloric intake.

Literature exploring the physiology underlying the refeeding syndrome, reviewed by Kohn, Madden and Clarke [8], highlighted disturbances of insulin and phosphate as central to this process. The refeeding syndrome is thought to be instigated by the influx of enteral glucose during refeeding causing an insulin surge and driving glucose, fluid and electrolytes into the intracellular space. This combined with the breakdown of the sodium/potassium pump regulating the intracellular environment due to a lack of phosphated adenosine to provide energy to the pump results in severe electrolyte disturbances, hypophosphatemia, cardiac failure and death [14]. Based on this understanding, it has been argued that restricting the percentage of daily energy provided by carbohydrate rather than total daily energy intake, in addition to the provision of supplemental phosphate, may be more effective in mitigating the risk of refeeding syndrome than reducing calories [8].

The starvation state is associated with low basal levels of insulin and high glucagon levels. It is hypothesised that this results in a delayed insulin response to refeeding, resulting in clinically significant post-prandial hypoglycaemia during early refeeding [14,16-19]. A study by Hart et al., [20] reported that $29 \%$ of adult patients with AN experienced post-prandial hypoglycaemia in the first weeks of refeeding with the highest rates occurring between one and three hours post meal. In 2011, Kohn et al., [8] proposed the use of continuous nasogastric refeeding during early refeeding to eliminate hypoglycaemia.
Clinical settings using current feeding guidelines have reported poor weight gain or weight loss in hospitalised adolescents with AN in the first week of admission resulting in further deterioration in nutritional status [21]. Six recently published studies, four retrospective chart reviews and two prospective cohort studies [15,21-25] have concluded that higher initial refeeding rates lead to faster weight gain, more rapid medical stabilisation and shorter lengths of hospital stay without adverse consequences.

The aim of the current study is to report refeeding outcomes for a cohort of adolescent patients with AN using a standardised refeeding regime involving supported oral meals and nasogastric feeding with sufficient calories to initiate and sustain weight gain from the outset of treatment. The proposed refeeding protocol aimed to address the risks associated with both the refeeding syndrome, poor weight gain and delayed medical recovery by limiting energy provided as carbohydrate to a maximum of $50 \%$ while providing sufficient energy to exceed total daily energy requirements. All patients were commenced on oral phosphate supplementation (500 mg Sandoz phosphate, twice daily). Additionally, the current protocol was designed to eliminate postprandial hypoglycaemia through continuous nocturnal NG feeds, a practice well tolerated by patients [26].

We hypothesized that the presented protocol would be well tolerated, promote timely weight restoration and not result in the occurrence of the refeeding syndrome.

\section{Methods}

This study was carried out in two specialist paediatric eating disorder services located in adjacent university teaching hospitals (The Sydney Children's Hospital Network, Westmead Campus (SCHN-W) and Westmead Hospital (WH)). A standard refeeding protocol, described in Figure 1, was followed at both treatment sites and compared to assess reliability.

\section{Participants}

This study cohort included all patients in a large randomised controlled inpatient treatment trial [27]. Participants were recruited from 266 consecutive eating disorder admissions to two specialist paediatric medical units between June 2007 and February 2010. Participants were eligible if they were aged between 12 and 18 years, with a DSM-IV diagnosis of AN of less than 3 years duration [28] and were medically unstable (hypothermic (temperature $<35.5^{\circ} \mathrm{C}$ ), bradycardic (heart rate $<50$ beats/min), hypotensive (blood pressure $<80 \mathrm{~mm} \mathrm{Hg}$ systolic and $<40 \mathrm{~mm} \mathrm{Hg}$ diastolic), orthostatic instability (pulse increase $>20$ beats $/ \mathrm{min}$, systolic blood pressure decrease $>20 \mathrm{~mm} \mathrm{Hg}$ ) or QT interval corrected for heart rate $>0.45$ s) [3]. All participants subsequently would 


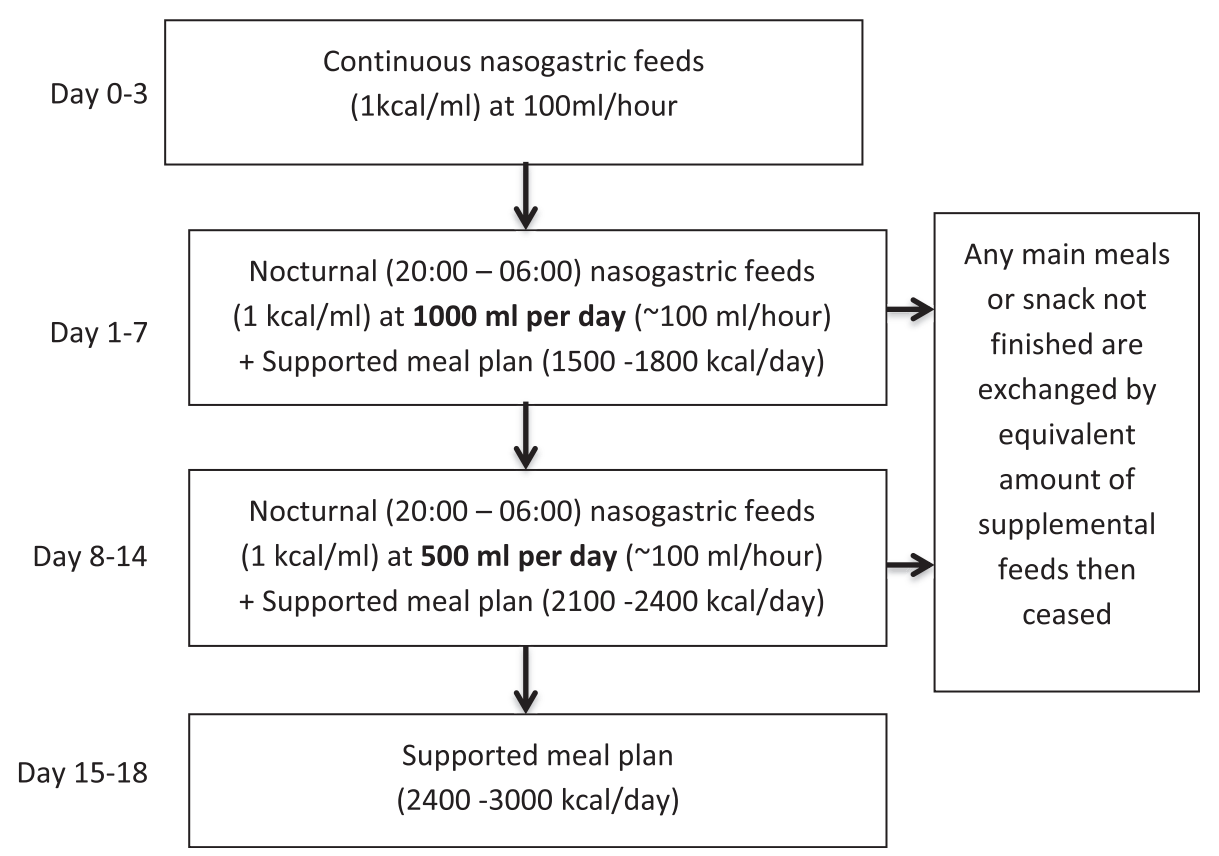

Figure 1 Refeeding protocol used in the nutritional rehabilitation of patients.

have met DSM 5 criteria for AN [29]. Eighty-five patients met trial criteria and 82 patients and their families consented to the research. Four patients withdrew from the trial during inpatient treatment, leaving 78 patients completing the inpatient treatment refeeding protocol at a $95 \%$ retention rate.

\section{Assessments and procedures}

Medical assessments were conducted by paediatricians experienced in the management of eating disorders and psychiatric assessments by an experienced child psychiatrist. Assessment included diagnostic evaluation for eating disorder symptomatology using medical and psychiatric assessment and standardised clinical interviews and questionnaires including the Eating Disorder Examination adult, youth and child versions [30-32].

\section{Medical assessment}

Body weight was recorded at intake, then bi-weekly for the duration of the admission. Body weight was recorded to the nearest $0.05 \mathrm{~kg}$ using a platform scale (A\&D FV-150 K) before breakfast, post-voiding, in a hospital gown and without footwear. Height was measured to the nearest $0.1 \mathrm{~cm}$ with a stadiometer (TB I Hyssna AB). Percent Expected Body Weight (\%EBW) was calculated using the $50^{\text {th }}$ percentile on tables for weight and height by age and gender using the Centre for Disease Control and Prevention standardised growth charts [33] (\%EBW = $\mathrm{BMI} / 50^{\text {th }}$ percentile $\mathrm{BMI}$ for age, height and gender $\times$ 100) [34].
To assess medical stability, heart rate (per minute) was reported as three times the recorded value for radial pulse measured for 20 seconds. Temperature was taken using a Becton and Dickinson electronic thermometer placed in the axilla. Blood pressure was recorded using a cuff covering two-thirds of the length of the right arm connected to a Space Lab Medical SL electronic sphygmomanometer. Participants were initially placed on 24 hour continuous cardiac monitoring with pulse rate, blood pressure and temperature recorded two-hourly. Once criteria for medical instability were no longer met during the day, study participants had four-hourly monitoring of pulse rate, blood pressure and temperature monitoring during the day, but remained on continuous overnight monitoring between 22:00 and 06:00 hours. Patients were examined on a daily basis for complications of refeeding syndrome including cardiac failure, dependent oedema and delirium.

Morning blood samples were taken to track blood electrolytes including phosphate and blood glucose levels. Blood samples were taken daily for the first 7 days and then twice weekly. Urinalysis, looking at specific gravity as a measure of hydration, was taken at admission and bi-weekly in conjunction with weights for the duration of admission.

\section{Measures}

The primary outcome measure was change in \%EBW at week 1, week 2 and 2.5 weeks. Secondary outcome measures included hypophosphatemia, hypoglycaemia, electrolyte abnormalities and clinical stigmata of the 
refeeding syndrome (cardiac failure, dependent oedema and delirium).

\section{Treatment}

Patients were refed using a standardised protocol commencing with 24 to 72 hours of continuous nasogastric feeds, ceased with day time medical stability, followed by a combination of nocturnal feeds and an oral meal plan aiming for a total caloric intake of between 2400$3000 \mathrm{kcal} /$ day. Nasogastric feeds were limited to $\leq 50 \%$ of energy from carbohydrates with approximately $30 \%$ energy from fats. Patients were prescribed routine phosphate supplements (Sandoz Phosphate, $500 \mathrm{mg}$, twice daily) from the commencement of treatment. The amount and duration of nasogastric feeding was determined by markers of medical instability. The total amount of prescribed calories was calculated to result in a weekly weight gain of $1 \mathrm{~kg}$ (see Figure 1). Fluid intake was limited to that provided by the nasogastric feeds and that prescribed in the oral menu plan and varied between $1540 \mathrm{ml}$ and $1920 \mathrm{ml}$ per day.

\section{Ethical approval}

This study was approved by the Human Research Ethics Committee of the Sydney Children's Hospital Network,
Westmead Campus, and Westmead Hospital (2006/114). Written consent was obtained from all participants and their families at hospital admission.

\section{Statistical analysis}

To assess group differences between the two treatment sites, independent t-tests were used. Repeated-measures analysis of variance was used to compare weight across time points. The criterion used for statistical significance was $p<0.05$ in two-tailed tests. Analyses were performed using SPSS version 21 for Mac.

\section{Results}

Fifty-two participants (67\%) were treated at SCHN-W and 26 participants $(33 \%)$ at WH. Participants were mostly female (95\%), aged $12-18$ years with a mean age of 14.84 years $(S D=1.46)$. The only statistically significant difference on baseline variables between sites was age at admission [SCHN-W $(M=14.00, S D=.99)$ vs. WH $(M=16.17, S D=1.05, t(76)=-9.24, p<.05)$ which was to be expected due to the different admission age criteria for each unit. There were no differences in illness history, clinical factors, or length of inpatient treatment between groups (Table 1). The protocol was

Table 1 Demographic and clinical characteristics of sample

\begin{tabular}{|c|c|c|c|}
\hline \multicolumn{4}{|c|}{$\mathrm{N}(\%)^{\mathrm{a}}$ or $M(S D)^{b}$} \\
\hline \multicolumn{4}{|l|}{ Demographic characteristics } \\
\hline & SCHN-W & WH & Total \\
\hline Sample Size & 52 & 26 & 78 \\
\hline Age (years)* & $14.00(.99)$ & $16.17(1.05)$ & $14.84(1.46)$ \\
\hline Gender - Male & $1(1.92)$ & $3(11.54)$ & $4(5.13)$ \\
\hline \multicolumn{4}{|l|}{ Ethnicity } \\
\hline White & $43(82.69)$ & $22(84.62)$ & $65(83.33)$ \\
\hline Asian & $9(17.31)$ & 0 & $9(11.54)$ \\
\hline Other & 0 & $4(15.38)$ & $4(5.13)$ \\
\hline \multicolumn{4}{|l|}{ Clinical characteristics at admission } \\
\hline \multicolumn{4}{|l|}{ Anorexia Nervosa subtype } \\
\hline Restricting only & $36(69.23)$ & $17(65.38)$ & $53(67.95)$ \\
\hline Restricting with bulimic features & $16(30.77)$ & $9(34.62)$ & $25(32.05)$ \\
\hline Excessive exercise & 19 (36.54) & $10(38.46)$ & $29(37.18)$ \\
\hline Duration of illness (months) & $6.56(4.37)$ & $9.27(7.97)$ & $7.60(6.16)$ \\
\hline Previous hospitalizations & $4(7.69)$ & $1(3.85)$ & $5(6.41)$ \\
\hline Percent expected body weight & 78.88 (6.13) & $77.54(7.09)$ & $78.37(6.50)$ \\
\hline Eating disorder examination global score & $3.04(1.12)$ & $2.99(1.13)$ & $3.02(1.12)$ \\
\hline \multicolumn{4}{|l|}{ Treatment characteristics } \\
\hline Length of admission (days) & $30.27(16.24)$ & $25.97(13.46)$ & $28.62(15.28)$ \\
\hline
\end{tabular}

${ }^{a}$ Chi-square test for independence was conducted to compare group differences for categorical variables and no statistically significant differences were found at the .05 level. ${ }^{b}$ Independent-samples t-test was conducted to compare trial group mean differences for each continuous variable and no statistically significant differences were found at the .05 level unless otherwise noted. *Statistically significant difference at the $p<.05$ level. 
consistently adhered to by all participants included in the analysis.

The mean \%EBW on admission was $78.37(S D=6.50)$. Paired-samples t-test revealed that there was a statistically significant gain in weight from admission to the end of the refeeding protocol for the cohort as a whole, with \%EBW at 2.5 weeks on average 85.58 [ $S D=6.54$, $t(77)=-17.02, p<0.05$, eta squared $=.79$ (very large effect)]. Weight gain in the first week of admission was at a mean of $2.79 \mathrm{~kg}(S D=1.27)$ with gains continuing throughout the 2.5 week admission $(M=5.12 \mathrm{~kg}, S D=$ 2.96). Statistically significant weight gain was seen from the end of the refeeding protocol to 12-month posttreatment follow-up where \%EBW was $95.33[\mathrm{SD}=$ 9.47, $\mathrm{t}(77)=-14.30, p<0.05$, eta squared $=.73$ (very large effect)].

Although there was a significant difference in weight $(\mathrm{kg})$ gained between sites at 0.5, 1, 1.5, 2 and 2.5 weeks, when weight was related to age, gender and height (i.e., transformed to \%EBW), there was no significant difference in changes in \%EBW between sites (see Table 2). The significant difference in weight gain when measured in kilograms relates to the significant difference in patient age between the two services. There was no significant difference in weight gain measured in kilograms based on AN subtype (Restricting: $n=54$; $M=3.88, S D=1.95$; Binge/Purge: $n=24, M=3.45$, $S D=1.95 ; t(77)=.915, p=.363, \mathrm{NS})$.

Serum phosphate and blood glucose levels were measured daily for a minimum of 7 days and then twice weekly once stable. No patient developed hypophosphatemia $(<1.0 \mathrm{mmol} / \mathrm{L})$, or hypoglycaemia $(<3.0 \mathrm{mmol} / \mathrm{L})$ at any point during treatment (see Figure 2 ).

All patients were examined medically on a daily basis during the course of their admission. No patient had an adverse reaction to the refeeding protocol nor developed any clinical signs or symptoms of the refeeding syndrome. In particular no patient developed signs of cardiac failure, dependent oedema or delirium.

\section{Discussion}

The refeeding guidelines proposed by this study (Figure 1) promote the provision of sufficient caloric intake to meet metabolic needs and facilitate weight gain from the outset of refeeding. The proposed starting caloric intake and rate of increase in caloric prescription exceeds that of current guidelines $[1,3,6]$. Patients treated with the study refeeding protocol safely and significantly gained weight over the course of treatment including in the first week of admission, without incidence of refeeding syndrome (cardiac failure, dependent oedema or delirium) or other complications, including hypophosphatemia or post-prandial hypoglycaemia. In addition to early weight gain the group showed ongoing weight gain to 12-month follow up with average \%EBW (95.33) consistent with definitions of weight recovery [35].

Weight gain within the first week of refeeding averaged $2.79 \mathrm{~kg}$. While this rate of weight gain is significantly in excess of what would be expected based on the prescribed caloric intake and in excess of current guideline recommendations $[1,7]$, with ongoing nutritional rehabilitation, weight gain averaged $1.34 \mathrm{~kg}$ per week during the first 2.5 weeks of admission. This was consistent with the theoretical increase from the total calories provided and was consistent with previous outcomes for patients receiving $2700-3000 \mathrm{kcal}$ each day [15]. All patients had base-line body composition studies using dual $\mathrm{x}$-ray absorptiometry (DXA), however, radiation exposure associated with this method precluded its use to measure changes in body composition over the period of this study. Previous research by our group has shown that other methods for measuring body composition

Table 2 Weight change over the course of admission

\begin{tabular}{|c|c|c|c|c|c|c|c|}
\hline & \multicolumn{7}{|l|}{$M(S D)$} \\
\hline & \multicolumn{3}{|c|}{ \%EBW per assessment } & & \multicolumn{3}{|c|}{ Weight gain per assessment (kg) } \\
\hline & SCHN-W & WH & Total & & SCHN-W & WH & Total \\
\hline Sample size & 52 & 26 & 78 & Sample size & 52 & 26 & 78 \\
\hline Admission & $78.88(6.13)$ & $77.54(7.09)$ & $78.37(6.50)$ & Admission Weight & $39.57(4.89)$ & $43.26(6.30)$ & $40.99(5.72)$ \\
\hline Week 0.5 & $82.93(6.98)$ & $80.13(6.66)$ & $81.85(6.95)$ & Week $0.5^{*}$ & $2.01(1.31)$ & $1.42(.83)$ & $1.78(1.78)$ \\
\hline Week 1 & $84.80(6.45)$ & $82.19(6.59)$ & $83.80(6.59)$ & Week 1 & $.93(.92)$ & $1.14(.60)$ & $1.01(.81)$ \\
\hline Week 1.5 & $85.62(6.51)$ & $83.42(6.32)$ & $84.77(6.49)$ & Week $1.5^{*}$ & $.41(.54)$ & $.68(.64)$ & $.51(.59)$ \\
\hline Week 2 & $85.88(6.66)$ & $84.08(6.28)$ & $85.19(6.54)$ & Week $2^{*}$ & $.13(.45)$ & $.36(.51)$ & $.22(.48)$ \\
\hline Week 2.5 & $85.90(6.49)$ & $85.06(6.48)$ & $85.58(6.46)$ & Week $2.5^{*}$ & $.01(.62)$ & $.54(.683)$ & $.22(.62)$ \\
\hline Total Gain & $7.02(3.21)$ & $7.52(4.42)$ & $7.21(3.70)$ & Total Gain & $4.98(2.37)$ & $5.34(3.75)$ & $5.12(2.96)$ \\
\hline 12 month FU & $94.90(10.21)$ & $93.94(7.87)$ & $94.53(9.33)$ & 12 month FU Weight & $51.59(5.79)$ & $56.00(7.44)$ & $53.00(6.64)$ \\
\hline
\end{tabular}




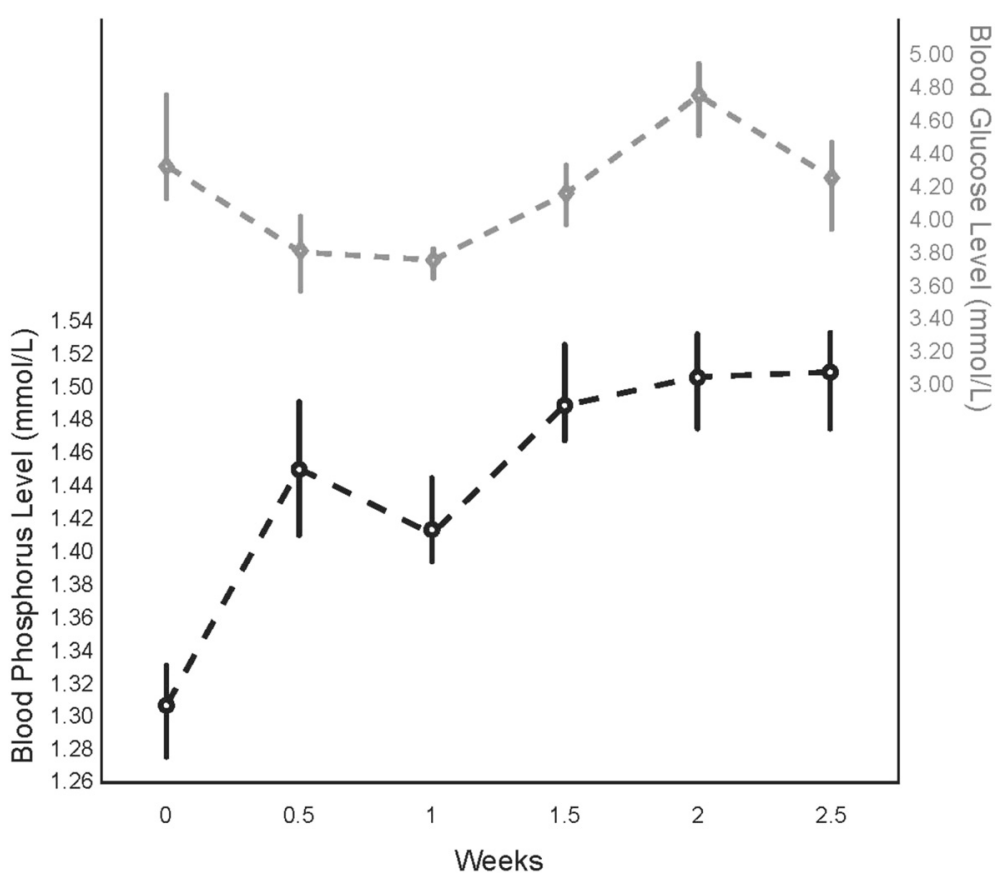

Figure 2 Blood levels with weight gain.

(bioelectrical impedance and skin fold thickness) to be inaccurate on an individual level and with wide levels of variation between results obtained for different methods $[36,37]$ and for this reason were not used to measure changes in body composition over this period. Physical examination and regular urinalysis did not reveal evidence of over-hydration in this group but it is likely that a portion of the weight gain in week one was due to rehydration. Research has shown that reestablishment of glycogen stores in the liver may account for significant weight gain with glycogen itself accounting for $400 \mathrm{~g}$ in healthy adults [38] and water bound to glycogen up to $1.2 \mathrm{~kg}$ [39].

The early weight gain in this study contrasts with treatment centres following currently published guidelines who universally report a decrease in weight during the first week of refeeding [21]. In the case of malnourished AN patients presenting with medical instability, the provision of insufficient calories to meet resting energy expenditure has been shown to prolong medical instability and the length of hospitalisation in this patient group [15,21-25]. There was no significant difference in weight gain between treatment sites when weight was related to gender, age and height, suggesting the replicability of this protocol.

Clinically significant post-prandial hypoglycaemia during initial refeeding of patients with AN has been reported in the literature [14,16-18]. In the current study, continuous nocturnal nasogastric tube feeding was used to address post-prandial hypoglycaemia by maintaining a constant level of available calories to maintain blood glucose levels and promote the restoration of tissue glycogen. Though it was beyond the scope of this study to systematically measure post-prandial blood glucose levels at all meals, no hypoglycaemia was observed in morning blood glucose measurements, taken 60-150 minutes after breakfast.

Avoidance of carbohydrate as the predominant macronutrient energy source was undertaken in view of previous observations that high carbohydrate loads predispose to hypophosphatemia and the refeeding syndrome [14]. The proposed protocol limited the proportion of carbohydrates to $<50 \%$ of dietary energy intake. In addition, oral phosphate supplements (500 mg, twice daily) were given during the study period. Hypophosphatemia was not seen in this patient group.

Psychological outcomes in this group have been previously published demonstrating low patient drop out rates during inpatient refeeding (4 out of 82 patients (4.9\%)) and significant improvements in eating disorder psychopathology at 12-month follow-up as measured by Eating Disorder Examination global score (Admission: $\mathrm{M}=3.03, \mathrm{SD}=1.13$ vs. 12 month follow up: $\mathrm{M}=1.59$, $\mathrm{SD}=1.36, \mathrm{t}(68)=7.88, p<0.05$, eta squared $=.49$ (very large effect)) [27]. Research published by this group has shown this protocol, in particular nasogastric refeeding, to be well tolerated in adolescents with AN [26] These findings are consistent with published research which has not demonstrated negative psychological outcomes with more rapid weight gain associated with nasogastric 
refeeding [40], high energy supplements [41] and oral refeeding [42] including weekly rates of weight gain in excess of $2 \mathrm{~kg}$ [42].

The protocol developed for this study arose from the need to have a standardised treatment regimen for this multi-centre randomised controlled inpatient treatment trial of medically unstable adolescents with AN [27]. The refeeding protocol was developed to better meet physiological need, to improve safety, and avoid refeeding syndrome. The described protocol provides important details, directions and outcomes about refeeding medically compromised adolescents with AN more rapidly than in current guidelines $[1,3,6]$. These results suggest that calorie prescription can safely exceed levels recommended in contemporary guidelines.

\section{Conclusions}

The refeeding protocol resulted in immediate weight gain and was tolerated with no indicators of refeeding syndrome. There were no significant differences in outcomes between the treatment sites, suggesting the protocol is replicable. The experience of using this refeeding protocol provides support for reviewing current recommendations for the treatment of medically unstable adolescents with Anorexia Nervosa.

\section{Abbreviations}

AN: Anorexia nervosa; SCHN-W: The Sydney children's hospital network (Westmead Campus); WH: Westmead hospital; \%EBW: Percent expected body weight.

\section{Competing interests}

The authors acknowledge the National Health and Medical Research Council of Australia (NHMRC) as the sole providers of funding for the study (Grant Identification Number 457235). The contents of the published material are solely the responsibility of the individual authors and do not reflect the views of the NHMRC.

Professor Phillipa Hay and Professor Stephen Touyz are the Editors-in-Chief of the Journal of Eating Disorders. Dr Sloane Madden is on the Editorial Board. The authors have no other conflicts of interest or additional financial disclosures to report.

\section{Authors' contributions}

SM participated in the design of the study, analysis and interpretation of the data and the drafting and revision of the manuscript. JM participated in the design of the study, the acquisition, analysis and interpretation of the data and was involved in the drafting and revision of the manuscript. SC participated in the design of the study, interpretation of the data, the revision of the manuscript. ST and PH participated in the discussion of the protocol and review of the manuscript. MK participated in the design of the study, interpretation of the data and the revision of the manuscript. All authors have given final approval for the version of the manuscript to be published and agree to be accountable for all aspects of the work in ensuring that questions related to the accuracy and integrity of any part of the work are appropriately investigated and resolved. SM (Primary author) and MK had full access to all of the data in the study and take responsibility for the integrity of the data and the accuracy of the data analysis.

\section{Authors' information}

Dr Madden (co-director), A/Prof Kohn (co-director) and Dr Miskovic-Wheatley are members of the Eating Disorder Service at The Sydney Children's Hospitals Network, Westmead Campus. A/Prof Clarke (director) and A/Prof Kohn are associated with the Centre for Research into Adolescents' Health
(CRASH), Adolescent Medicine Unit, Westmead Hospital. Dr Madden and A/ Prof Kohn are affiliated with the Discipline of Paediatrics, Faculty of Medicine; Prof Touyz the School of Psychology, and Dr Miskovic-Wheatley the Westmead Clinical School, at the University of Sydney. Prof Hay is from the School of Medicine, University of Western Sydney.

Although no data from this specific study has previously been published, the theoretical position to this study has been published and preliminary data presented at the following conferences and meetings:

Title: Safe and efficient refeeding in adolescents admitted to hospital with anorexia nervosa.

1. Society for Adolescent Health and Medicine, Annual Meeting, New Orleans, 2012

2. Academy of Eating Disorders, International Conference on Eating

Disorders, New York, 2014

The main trial from which this study is drawn is reported in the related publication:

Madden S, Miskovic-Wheatley J, Wallis A, Kohn M, Lock J, Le Grange D, Jo B, Clarke S, Rhodes P, Hay P, Touyz S. A randomised controlled trial of inpatient treatment for anorexia nervosa in medically unstable adolescents. Psychol Med 2014; FirstView:1-13. DOI:10.1017/S0033291714001573.

\section{Acknowledgements}

Thank you to medical student Ilyn P. de Leon for her invaluable work with the data collection, initial analysis and preliminary interpretation. The authors gratefully acknowledge the tireless work of the clinical nursing staff of the inpatient programs of The Sydney Children's Hospitals Network, Westmead Campus and Westmead Hospital, led by Clinical Nurse Consultants Joanne Titterton (MCliPra (Paed), UTS) and Gail Anderson (MN, UTS). Thank you to all the young people and their families who participate in clinical research programs. Without such participation, the field could not continue in the pursuit of service improvement and illness reduction.

\section{Author details}

'Eating Disorder Service, The Sydney Children's Hospitals Network, Westmead Campus, Locked Bag 4001, Westmead 2145, NSW, Australia. ${ }^{2}$ Discipline of Psychiatry, Faculty of Medicine, The University of Sydney, Sydney, Australia. ${ }^{3}$ Westmead Clinical School, The Sydney Children's Hospitals Network, Westmead Campus, Sydney, Australia. ${ }^{4}$ Discipline of Paediatrics, Faculty of Medicine, University of Sydney, Sydney, Australia. ${ }^{5}$ Centre for Research into AdolescentS' Health (CRASH), University of Sydney, Sydney, Australia. ${ }^{6}$ Clinical Psychology Unit, University of Sydney, Sydney, Australia.

${ }^{7}$ School of Medicine, University of Western Sydney, Sydney, Australia.

Received: 12 November 2014 Accepted: 28 February 2015

Published online: 25 March 2015

\section{References}

1. American Psychiatric Association. Treatment of patients with eating disorders (3rd ed.). Am J Psychiat. 2006;163(7 Suppl):4-54.

2. Beumont PJ, Large M. Hypophosphatemia, delirium and cardiac arrhythmia in anorexia nervosa. Med J Australia. 1991;155(8):519-22.

3. Golden ND, Katzman DK, Kreipe RE, Stevens SL, Sawyer SM, Rees J, et al. Eating disorders in adolescents: position paper of the society for adolescent medicine. J Adolesc Health. 2003;33(6):496-503.

4. Gentile MG, Pastorelli P, Ciceri R, Manna G, Collimedaglia S. Specialized refeeding treatment for anorexia nervosa patients suffering from extreme undernutrition. Clin Nutr. 2010;29(5):627-32. doi: 10.1016/j.clnu.2010.03.008.

5. Rocks T, Pelly F, Wilkinson P. Nutrition therapy during initiation of refeeding in underweight children and adolescent inpatients with anorexia nervosa: a systematic review of the evidence. J Acad Nutr Diet. 2014;114(6):897-907. doi: 10.1016/j.jand.2013.11.022.

6. National Collaborating Centre for Mental Health (2004). Eating disorders: Core interventions in the treatment and management of anorexia nervosa, bulimia nervosa and related eating disorders. NICE Clinical Guidelines, No. 9. Leicester, UK: British Psychological Society; 2004.

7. Hay P, Chinn D, Forbes D, Madden S, Newton R, Sugenor L, et al. Royal Australian and New Zealand College of Psychiatrists clinical practice guidelies for the treatment of eating disorders. Aust N Z J Psychiatry. 2014;48:997-1009. doi: 10.1177/0004867414555814.

8. Kohn MR, Madden S, Clarke S. Refeeding in anorexia nervosa: increased safety and efficiency through understanding the pathophysiology of protein 
calorie malnutrition. Curr Opin Pediatr. 2011;23(4):390-4. doi: 10.1097/ MOP.0b013e3283487591.

9. Weinsier R, Krumdieck C. Death resulting from overzealous total parenteral nutrition: the refeeding syndrome revisited. Am J Clin Nutr. 1981;34(3):393-9.

10. Sheridan PH, Collins M. Potentially life-threatening hypophosphatemia in anorexia nervosa. J Adolesc Health Care. 1983:4(1):44-6. doi: 10.1016/ S0197-0070(83)80228-9.

11. Schoken D, Holloway J. Weight loss and the heart: effects of anorexia nervosa and starvation. Arch Intern Med. 1989;149(4):877-81. doi: 10.1001/ archinte. 1989.00390040085017.

12. Kohn MR, Golden NH, Shenker IR. Cardiac arrest and delirium: presentations of the refeeding syndrome in severely malnourished adolescents with anorexia nervosa. J Adolesc Health. 1998;22(3):239-43. doi: 10.1016/ S1054-139X(97)00163-8.

13. Ornstein RM, Golden N, Jacobson MS, Shenker IR. Hypophosphatemia during nutritional rehabilitation in anorexia nervosa: implications for refeeding and monitoring. J Adolesc Health. 2003;32(1):83-8. doi: 10.1016/ S1054-139X(02)00456-1.

14. O'Connor G, Goldin J. The refeeding syndrome and glucose load. Int J Eat Disord. 2010;44:182-5. doi: 10.1002/eat.20791.

15. Whitelaw M, Gilbertson H, Lam P-Y, Sawyer S. Does aggressive refeeding in hospitalized adolescents with anorexia nervosa result in increased hypophospataemia? J Adolesc Health. 2010;46(6):577-82. doi: 10.1016/j. jadohealth.2009.11.207.

16. Gniuli DE, Liverani E, Capristo E, Greco A, Mingrone G. Blunted glucose metabolism in anorexia nervosa. Metabolism. 2001;50(8):876-81. doi: 10.1053/meta.2001.24915

17. Yasuhara D, Naruo T, Nagai N, Muranaga T, Nakhara T, Tanaka M, et al. Glucose tolerance predicts short-term refeeding outcome in females with anorexia nervosa. Psychosom Med. 2005;67(4):669-76.

18. Kinzig KJ, Coughlin JW, Redgrave GW, Moran T, Guarda A. Insulin, glucose, and pancreatic polypeptide responses to a test meal in restricting type anorexia nervosa before and after weight restoration. Am J Physiol Endocrinol Metab. 2007;292(5):E1441-6. doi: 10.1152/ajpendo.00347.2006.

19. Hart $\mathrm{S}$, Abraham $\mathrm{S}$, Luscombe $\mathrm{G}$, Russell J. Eating disorder management in hospital patients: current practice among dietitians in Australia. Nutr Diet. 2008;65(1):16-22. doi: 10.1111/j.1747-0080.2007.00174.x.

20. Hart S, Abraham S, Franklin R, Russell J. Weight changes during inpatiet refeeding of underweight eating disorder patients. Eur Eat Disord Rev. 2011;19(5):390-7. doi: 10.1002/erv.1052.

21. Garber AK, Michihata N, Hetnal K, Shafer M, Mosicki A. A prospective examination of weight gain in hospitalized adolescents with anorexia nervosa on a recommended refeeding protocol. J Adolesc Health. 2012;50(1):24-9. doi: 10.1016/j.jadohealth.2011.06.011.

22. Garber AK, Mauldin K, Michihata N, Buckelew S, Shafer MA, Moscicki AB. Higher calorie diets increase rate of weight gain and shorten hospital stay in hospitalized adolescents with anorexia nervosa. J Adolesc Health. 2013;53(5):579-84. doi: 10.1016/j.jadohealth.2013.07.014

23. Golden NH, Keane-Miller C, Sainani KL, Kapphahn CJ. Higher caloric intake in hospitalized adolescents with anorexia nervosa is associated with reduced length of stay and no increased rate of refeeding syndrome. J Adolesc Health. 2013;53(5):573-8. doi: 10.1016/j.jadohealth.2013.05.014.

24. Agostino H, Erdstein H, Di Meglio G. Shifting paradigms: continuous nasogastric feeding with high caloric intakes in anorexia nervosa. J Adolesc Health. 2013;53(5):590-4. doi: 10.1016/j.jadohealth.2013.06.005.

25. Leclerc A, Turrini T, Sherwood K, Katzman DK. Evaluation of a nutrition rehabilitation protocol in hospitalized adolescents with restrictive eating disorders. J Adolesc Health. 2013;53(5):585-9. doi: 10.1016/j. jadohealth.2013.06.001

26. Halse CD, Boughtwood D, Clarke S, Honey A, Kohn MR, Madden S. Illuminating multiple perspectives: meanings of nasogastric feeding in anorexia nervosa. Eur Eat Disord Rev. 2005;13(4):264-72. doi: 10.1002/erv.624.

27. Madden S, Miskovic-Wheatley J, Wallis A, Kohn M, Lock J, Le Grange D, et al. A randomised controlled trial of inpatient treatment for anorexia nervosa in medically unstable adolescents. Psychol Med. 2014;First View:1-13. doi: 10.1017/S0033291714001573.

28. American Psychiatric Association. Diagnostic and statistical manual of mental disorders (4th ed, text rev.). Washington, DC: American Psychatric Association; 2000.

29. American Psychiatric Association. Diagnostic and statistical manual of mental disorders (5th ed.). Arlington, VA: American Psychatric Association; 2013. ISBN 978-0-89042-554-1.
30. Fairburn C, Beglin S. The assessment of eating disorders: interview or self-report questionnaire? Int J Eat Disorder. 1994;16(4):363-70. doi: 10.1002/ 1098-108X(199412)16:4<363::AID-EAT2260160405>3.0.CO;2-\#.

31. Goldschmidt AB, Doyle AC, Wilfley DE. Assessment of binge eating in overweight youth using a questionnaire version of the child eating disorder examination with instructions. Int J Eat Disorder. 2007;40(5):460-7. doi: 10.1002/eat.20387.

32. Bryant-Waugh RJ, Cooper PJ, Taylor CL, Lask BD. The use of the eating disorder examination with children: a pilot study. Int J Eat Disorder. 1996;19(4):391-7. doi: 10.1002/(SICI) 1098-108X(199605)19:4<391:AID-EAT6>3.0. $\mathrm{CO} ; 2-\mathrm{G}$.

33. Kuczmarski RC, Ogden CL, Guo SS, Grummer-Strawn L, Flegal K, Mei Z, et al. 2000 growth charts for the United States: Methods and development, Vital and Health Statistics, Series 11, Data from the National Health Survey. 246th ed. 2002. p. 1-190

34. Le Grange D, Doyle PM, Swanson SA, Ludwig K, Glunz C, Kreipe RE. Calculation of expected body weight in adolescents with eating disorders. Pediatrics. 2012;129(2):438-46. doi: 10.1542/peds. 2011-1676.

35. Couturier J, Lock J. What is recovery in adolescent anorexia nervosa? Int J Eat Disord. 2006;39(7):550-5. doi: 10.1002/eat.20309.

36. Haas VK, Allen JR, Kohn MR, Clarke SD, Zhang S, Briody JN, et al. Total body protein in healthy adolescent girls: validation of estimates derived from simpler measures with neutron activation analysis. Am J Clin Nutr. 2007:85(1):66-72.

37. Haas VK, Kohn MR, Clarke SD, Allen JR, Madden S, Muller MJ, et al. Body composition changes in female adolescents with anorexia nervosa. Am J Clin Nutr. 2009;89(4):1005-10. doi: 10.3495/ajcn.2008.26958.

38. Kreitzman SN, Coxon AY, Szaz KF. Glycogen storage: illusions of easy weight loss, excessive weight regain, and distortions in estimates of body composition. Am J Clin Nutr. 1992;56(1 Suppl):292S-3.

39. King RF, Cooke C, Carroll S, O'Hara J. Estimating changes in hydration status from changes in body mass: considerations regarding metabolic water and glycogen storage. J Sports Sci. 2008;26(12):1361-3. doi: 10.1080/ 02640410802192768.

40. Robb AS, Silber TJ, Orrell-Valente JK, Valadez-Meltzer A, Ellis N, Dadson MJ, et al. Supplemental nocturnal nasogastric refeeding for better short-term outcome in hospitalized adolescent girls with anorexia nervosa. Am J Psychiatry. 2002;159(8):1347-53. doi: 10.1176/appi.ajp.159.8.1347.

41. Imbierowicz K, Braks K, Jacoby GE, Geiser F, Conrad R, Schilling G, et al. High-caloric supplements in anorexia treatment. Int J Eat Disord. 2002;32(2):135-45. doi: 10.1002/eat.10085.

42. Redgrave GW, Coughlin JW, Schreyer CC, Martin LM, Leonpacher AK, Seide $\mathrm{M}$, et al. Refeeding and weight restoration outcomes in anorexia nervosa: challenging current guidelines. Int J Eat Disord. 2015. doi: 10.1002/eat.22390.

\section{Submit your next manuscript to BioMed Central and take full advantage of:}

- Convenient online submission

- Thorough peer review

- No space constraints or color figure charges

- Immediate publication on acceptance

- Inclusion in PubMed, CAS, Scopus and Google Scholar

- Research which is freely available for redistribution

Submit your manuscript at www.biomedcentral.com/submit
C BioMed Central 